\title{
Vascular contributions to cognitive impairment
}

\author{
Clinton B. Wright, MD, MS
}

Alan Flores, MD

\begin{abstract}
Summary
Unlike many neurodegenerative causes of cognitive impairment and dementia, vascular damage is preventable. Despite the heterogeneity of vascular cognitive impairment (VCI) and the complexity of its clinical presentations, the potential for limiting progression and changing the trajectory of damage makes it all the more important for physicians to be educated about the syndrome and to remain vigilant when taking care of patients. In this review, we outline an approach to patients with possible $\mathrm{VCl}$, summarize current treatment and prevention guidelines, and provide an overview with case examples.
\end{abstract}
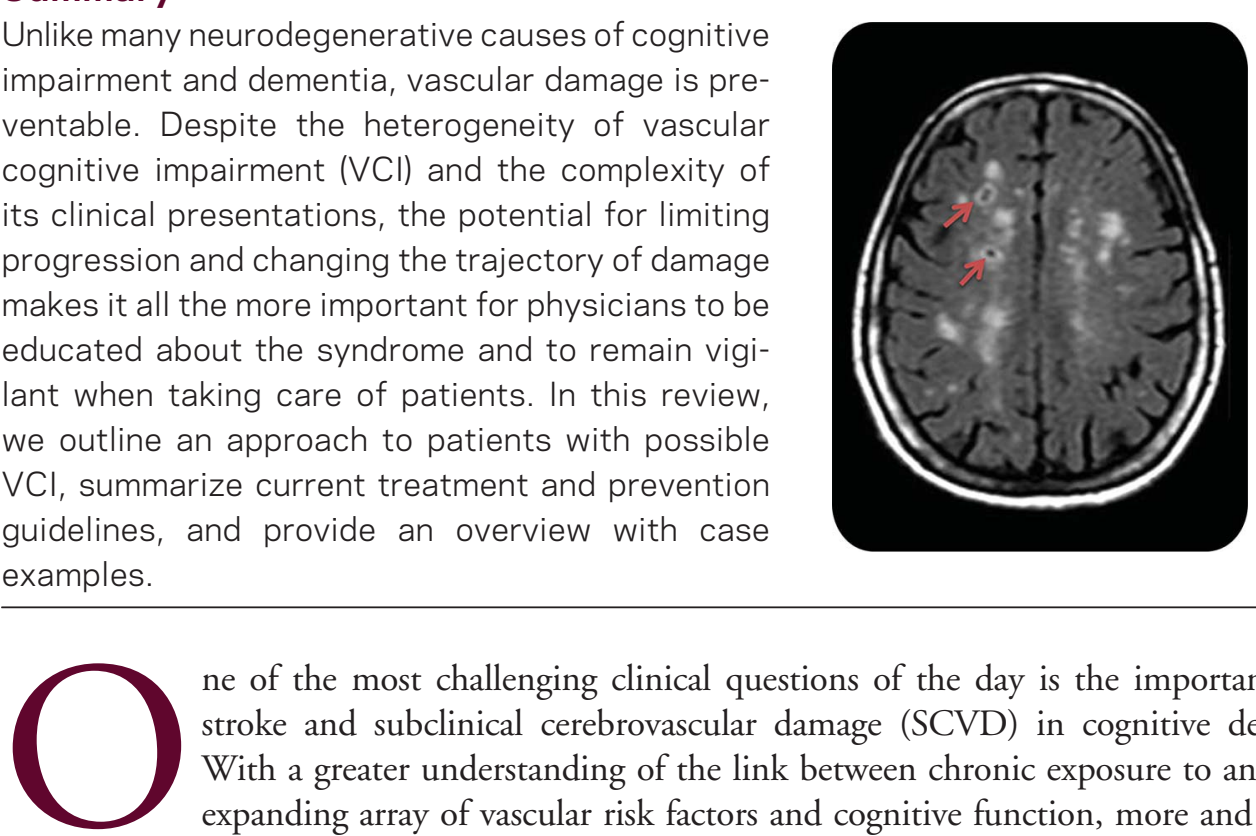

ne of the most challenging clinical questions of the day is the importance of stroke and subclinical cerebrovascular damage (SCVD) in cognitive decline. With a greater understanding of the link between chronic exposure to an everexpanding array of vascular risk factors and cognitive function, more and more clinicians are likely to consider vascular contributions to cognitive impairment-so-called vascular cognitive impairment (VCI) — that is, any type of cognitive impairment in which vascular disease plays a role. Several potentially modifiable risk factors for vascular disease have been shown to increase the risk of VCI, including hypertension, diabetes mellitus, dyslipidemia, and the metabolic syndrome, and these have been associated with an increased risk of dementia as well as Alzheimer disease (AD). ${ }^{1-3}$ The purpose of the current review is to provide an approach to the patient with either a history of clinical stroke or evidence of SCVD such as white matter lesions or imaging-defined infarctions.

Vascular dementia $(\mathrm{VaD})$ is second to $\mathrm{AD}$ as a cause of dementia in older adults, but the newly defined syndrome of VCI has broadened this definition to include a wide range of severities, from mild vascular cognitive impairment (VaMCI) to $\mathrm{VaD}$, and allows overlap with other cognitive disorders. As defined by the 2011 American Heart Association (AHS)/American Stroke Association (ASA) scientific statement on vascular contributions to cognitive

Evelyn F. McKnight Brain Institute and the Departments of Neurology, Public Health Sciences, and the Neuroscience Program (CBW), Leonard M. Miller School of Medicine, University of Miami, FL; and Department of Neurology, Vall d'Hebron University Hospital, Vall d'Hebron Research Institute and Department of Medicine (AF), Universitat Autonoma de Barcelona, Barcelona, Spain.

Funding information and disclosures are provided at the end of the article. Full disclosure form information provided by the authors is available with the full text of this article at Neurology.org/cp.

Correspondence to: c.wright21@med.miami.edu 


\section{It is important to consider the underlying etiologies of different vascular lesions once subclinical cerebrovascular damage has been detected with neuroimaging.}

impairment and dementia, VCI is any cognitive syndrome associated with vascular disease in which there is "demonstration of... a cognitive disorder by neuropsychological testing and either a history of clinical stroke or the presence of vascular disease by neuroimaging that suggests a link between the cognitive disorder and vascular disease." ${ }^{\prime 4}$ The statement created "possible" and "probable" categories that depend on the availability of data and the extent of competing pathologies.

\section{Mixed pathologies}

It is important for clinicians to be able to recognize $\mathrm{VCI}$ in its purest forms, such as multi-infarct dementia (MID), strategic-infarct dementia, and vaso-occlusive disease. However, large population-based autopsy studies have confirmed that the hallmarks of $\mathrm{AD}$ (amyloid plaques and neurofibrillary tangles) and other neurodegenerative conditions, such as Lewy body dementia, co-occur with vascular damage so frequently that mixed pathologies are probably more common than any individual entity. This co-occurrence, in addition to the lack of specificity of different clinical presentations for any single underlying pathology in the majority of cases, is why the definition of $\mathrm{VCI}$ has been broadened. A memory deficit is no longer required, allowing deficits in any cognitive domain. Patients with VCI may be more likely to present with deficits in executive function and psychomotor speed, but this is not always the case, and revised definitions of $\mathrm{AD}$ dementia now accept nonamnestic presentations (including executive dysfunction). ${ }^{5}$

\section{Common clinical presentations of $\mathrm{VCl}$}

General considerations A patient presenting with cognitive complaints who has longstanding hypertension or diabetes mellitus or a history of cardiac, peripheral artery, or chronic kidney disease may suggest possible VCI to one clinician, while to another, VCI may not be considered unless brain imaging shows vascular damage or there is a history of stroke. Thus, when to do an evaluation for VCI is a critical question without a clear answer (figure e-1 at Neurology.org/cp).

Vascular risk factors are often thought of in terms of their effect on the risk of clinical stroke, but many studies also associate them with markers of SCVD, including white matter lesions (WMLs), MRI-defined infarcts, microbleeds, and brain atrophy. These biomarkers, especially WMLs and MRI-defined infarcts, have been shown to predict outcomes that fall within the rubric of VCI, including mild cognitive impairment (MCI) and dementia. ${ }^{6}$ For this reason, any diagnostic workup of VCI necessarily includes structural brain imaging. The deciding factor for ordering neuroimaging in older adults who present with cognitive complaints may simply be a history of vascular risk factors, even in those whose primary complaint is trouble with memory. It is important to consider the underlying etiologies of different vascular lesions once SCVD has been detected with neuroimaging. For example, infarcts that appear to have been caused by emboli should prompt cardiac, extracranial, and intracranial ultrasound. And both small and large vessel infarcts should prompt a review of blood pressure, glycemic status, and fasting lipid levels. In people younger than 50 or 60 years who present with cognitive problems, other etiologies of VCI such as cerebral autosomal dominant arteriopathy with subcortical infarcts and leukoencephalopathy (CADASIL), Fabry disease, the type IV collagen mutations, and mitochondrial encephalopathy, lactic acidosis, and stroke-like episodes (MELAS) should be investigated. 
Neuropsychological profiles in VCI Another important issue in the evaluation of VCI is the common expectation that patients present primarily with attentional and executive control problems demonstrated by impairments in concentration or planning and carrying out daily activities. In general, it is important to keep in mind that there is substantial variability in the way patients present with cognitive problems, regardless of the underlying process. It is common to look for problems with executive control and attention in those with suspected VCI and problems with the memory domain in those with suspected $\mathrm{AD}$, but these are not mutually exclusive. ${ }^{7}$ While autopsy data suggest that patients presenting with cognitive impairment or dementia who are later found to have $\mathrm{AD}$ have worse memory than executive performance, those later found to have predominantly vascular pathology were more impaired on executive tests only two-thirds of the time. ${ }^{8}$ Further, these data showed that the dysexecutive syndrome was present in $10 \%$ of people later found to have $\mathrm{AD}$ on a pathologic basis. An appropriate clinical evaluation of several cognitive domains (executive/attention, visuospatial, language), including those testing cortical-subcortical connections, should be done.

In suspected cases of poststroke VCI, the clinician may perform bedside cognitive screening such as the Montreal Cognitive Assessment (MoCA; www.mocatest.org), using a cutoff score of less than 26 to define MCI. The Mini-Mental State Examination can be used, but it shows ceiling effects. ${ }^{9}$ More detailed cognitive testing can be considered should the MoCA be abnormal. The National Institute of Neurological Disorders and Stroke-Canadian Stroke Network vascular cognitive impairment harmonization standards provide both research and clinical batteries for use in the assessment of VCI. ${ }^{10}$

VCI after stroke Stroke is a potent risk factor for subsequent dementia, as shown by data from both hospital- and population-based studies and meta-analyses. ${ }^{11}$ Prospective observational studies have demonstrated a high incidence of cognitive impairment and dementia after stroke, with rates ranging from $6 \%$ to $32 \%$ in patients followed from 3 months to 20 years. ${ }^{12}$ Even lacunar infarcts presenting with classical syndromes, such as pure motor or pure sensory deficits, appear to associate strongly with poststroke cognitive impairment. ${ }^{13}$ In the Secondary Prevention of Small Subcortical Strokes (SPS3) trial, which included only patients with lacunar infarcts, $47 \%$ of participants met criteria for MCI between 2 weeks and 6 months (roughly half between 6 weeks and 3 months) poststroke. ${ }^{14}$ It is noteworthy that cognitive deficits were so common in the SPS3 trial even though the trial excluded those with severe disability. Indeed, deficits were seen among participants with no disability as measured by the modified Rankin scale. This is consistent with the idea that patients who have a first brain infarct may develop VCI even in the absence of a motor or sensory deficit. In such cases, a careful history with corroborating input from a reliable caregiver or informant will often reveal cognitive decline relative to prestroke abilities. However, such lesions are often "silent," in that they do not lead to a clinical evaluation of stroke, and the resulting cognitive impairment may be insidious. The patient should be examined carefully for focal motor signs, gait disturbance (magnetic gait, gait dyspraxia, parkinsonian gait), unsteadiness or frequent falls, urinary incontinence, and emotional incontinence (pseudobulbar palsy). Other bedside tests, such as the Frontal Assessment Battery, are fast, easy to administer, and sensitive to vascular damage. ${ }^{15}$

VCI without clinical stroke In the absence of clinically recognized stroke, the symptoms and signs suggestive of VCI are often less clear. Nevertheless, the presence of small vessel disease on pathologic examination was associated with a more than 2-fold increased risk of dementia at the age of 75 in the Medical Research Council Cognitive Function and Ageing Study. ${ }^{16}$ The clinical course can provide important information, as patients with VCI can have stepwise progression, and sudden decrements in cognitive function may occur in patients with strategic infarcts in the thalamus, anterior limb of the internal capsule, and, rarely, the hippocampus itself. The presence of focal neurologic signs may also help to make an accurate diagnosis of VCI (see appendix e-1, case example 1). The Hachinski Ischemic Score (HIS) is a tool that 


\section{While researchers have developed quantitative measures of WMLs, clinicians still rely on subjective readings by radiologists of the amount of damage.}

assigns points to these features and was developed to identify patients with MID. Pathology studies have shown that the HIS provides good discrimination between AD and MID. ${ }^{17}$ However, a meta-analysis showed that the HIS is not as helpful for discriminating between $\mathrm{AD}$ and mixed dementia. Some of the hallmarks of stroke previously thought to be markers of VCI, such as stepwise progression and abrupt onset of symptoms, have been found to be uncommon as well. ${ }^{18}$

White matter lesions Whatever the motivation for neuroimaging, one of the most common findings encountered by the evaluating clinician is varying amounts of WMLs (hypodensities on CT or hyperintensities on MRI). The wide variability in the extent of these lesions, even in cognitively normal elderly individuals, has left open the question of how much damage is both necessary and sufficient to cause VCI. While researchers have developed quantitative measures of WMLs, clinicians still rely on subjective readings by radiologists of the amount of damage. In the clinic it is common to be presented with reports that use vague terminology, making it difficult to know whether patients with "extensive" WMLs are adversely affected by them. Several visual rating scales to determine the burden and progression of WMLs on MRI have been used to stratify the risk, but these have variable interrater reliability and are not generally applied in the clinic.

It is also important to recognize special disease states suggested by abundant WMLs. The distribution and type of vascular lesions on imaging may help sort out the underlying etiology. For example, WMLs that extend into the poles of the temporal lobes and subinsular region are somewhat specific for CADASIL, and the presence of lobar microbleeds or a larger lobar hemorrhage suggests cerebral amyloid angiopathy (CAA). ${ }^{19,20}$ In such cases, other clues include the age at presentation (often younger in CADASIL and older than age 70 in CAA) and suggestive aspects of the medical history (e.g., migraine, seizures, and psychiatric disorders in CADASIL).

Subclinical infarcts or "silent strokes" Population-based studies from diverse communities around the world have found a high prevalence of subclinical infarction (SBI), and these lesions have been associated with incident cognitive decline and dementia. ${ }^{21}$ The cognitive effects of subcortical SBI are related to disruption of cortical-cortical and cortical-subcortical white matter tracts, but it is critical to keep in mind that small subcortical infarcts may have distant effects on cortical areas due to diaschisis. This hypothesis is supported by case series of CT-demonstrated subcortical infarcts with associated transcortical aphasias or neglect, as well as by functional studies using PET or SPECT. ${ }^{22}$

Another critical issue for the clinician confronted with neuroimaging showing possible infarction in the absence of a history of clinical stroke is whether the lesion is an infarct at all. A number of characteristics on MRI increase the probability that a lesion is a chronic infarct, such as the presence of a round or oval cavity on the T1 sequence, a hyperintense rim on the fluid-attenuated inversion recovery (FLAIR) sequence that corresponds with perilesional gliosis, and the absence of a flow void on the T2 sequence (figure 1A). However, in some cases, such as when lesions occur in the midst of extensive WMLs, some of these characteristics, such as evidence of gliosis, may be obscured (figure 1B). In cases of VCI, focal signs, such as motor weakness, that correlate with the location of the lesion are often not present, lowering the positive predictive value of the imaging finding. Another common misclassification made 
Figure Imaging markers of cerebrovascular damage

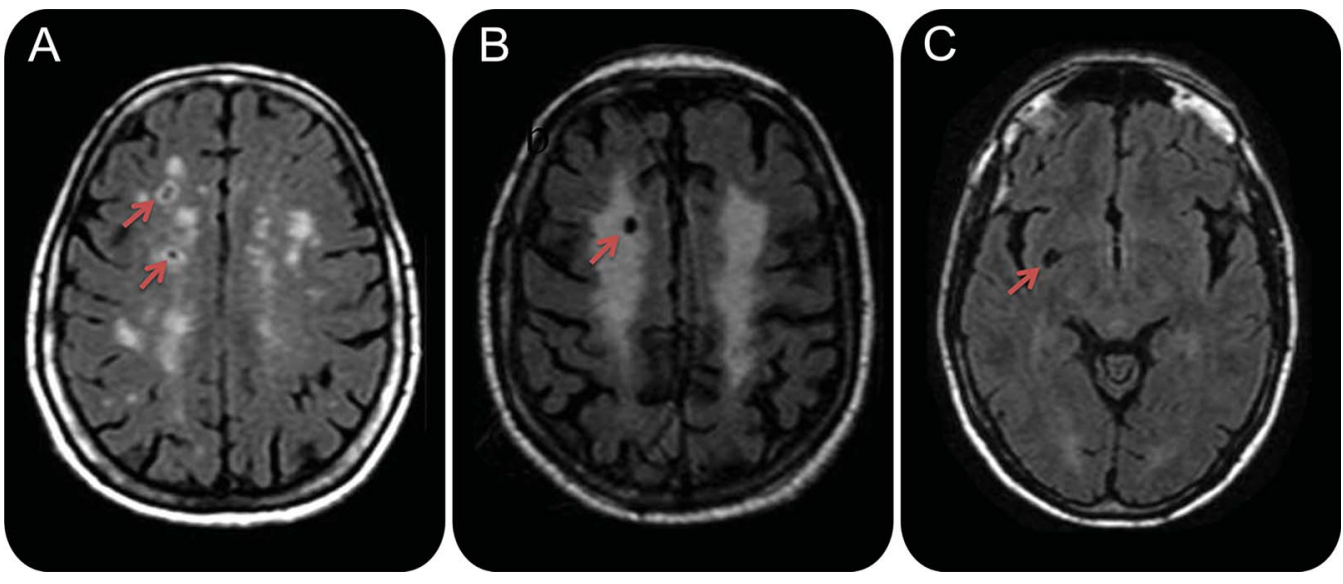

(A) Small subcortical lesions (arrows) with cavities and surrounding gliosis are probably chronic infarcts. (B) Subcortical lesions within areas of confluent hyperintensity are cystic in appearance and are also likely infarctions (arrow). (C) Infraputaminal lacunes occur lateral to the anterior commissure and are not infarcts (arrow), but their etiology is unclear and vascular disease may be a contributor.

even by experienced readers is calling a perivascular space (PVS) (or Virchow-Robin space) an infarct. There has been increasing interest in PVS, and evidence suggests that they may be related to hypertension and other vascular risk factors, but many are not infarcts. ${ }^{23}$ Characteristics that may distinguish PVS from infarcts include the presence of a flow void on T2 and symmetric lesions. And some PVS occur in typical locations; for example, an entity known as "infraputaminal lacune" can be a large PVS in the basal ganglia region and is often mistaken for an infarct (figure 1C). ${ }^{24}$ The recent Standards for Reporting Vascular Changes on Neuroimaging (STRIVE) have attempted to address some of these concerns by defining terms such as "lacune of presumed vascular origin" and expected findings. ${ }^{25}$ At the same time, it is important to keep in mind that microinfarcts are common and are generally not visible on clinical MRI scanners, even at 3T. Population-based autopsy data have shown that those with microinfarcts even without macroinfarcts have a greater risk of dementia. ${ }^{26}$

Mixed pathologies Patients often present with cognitive decline and imaging findings that are not specific (see appendix e -1 , case example 1). Imaging findings such as the presence of atrophy in the mesial temporal lobes, and to some extent global brain atrophy, often suggest $\mathrm{AD}$ to the clinician. However, the degree to which these changes are manifestations of SCVD that contribute to VaMCI is less clear. In addition, WMLs are seen as discrete or confluent, often bilateral and symmetrical, bright areas on T2 and FLAIR MRI sequences (or hypodensities on CT) and are a common radiologic finding in older adults. The number of these lesions has been strongly associated with age, with the reported prevalence in observational studies as high as $95 \%$ in those 60 years and older. ${ }^{27}$ Studies that have correlated pathology with neuroimaging have linked WMLs to microvascular damage and arteriolosclerosis, and WMLs have been ascribed to chronic hypoperfusion with resulting demyelination. However, other etiologies are plausible, including degenerative changes. For example, a recent population-based study from a sample in Northern Manhattan found that parietal WMLs were more predictive of incident $\mathrm{AD}$ than hippocampal volume. ${ }^{28} \mathrm{~A}$ somewhat separate issue from white matter hyperintensities having overlapping etiologies, such as $\mathrm{AD}$ and SCVD, is the synergistic effect of cerebral ischemia on AD pathophysiology. The Nun Study of Aging and Alzheimer's Disease has shown that less $\mathrm{AD}$ pathology is present at a given level of cognitive impairment when vascular damage is present.

Given the extensive overlap of vascular changes with other pathologic processes, ancillary testing may also be performed. Amyloid imaging with PET using the recently US Food and 


\section{Table Evidence for treatment of vascular dementia}

\section{Modality \\ Risk factor control}

Hypertension

Hyperlipidemia

Lifestyle

\section{Pharmacologic therapy}

Antiplatelet

Cholinesterase

inhibitors

\section{Summary of recommendations from AHA/ASA statement on $\mathrm{VCl}^{\mathrm{a}}$}

Stroke patients: Lowering blood pressure is effective for reducing the risk of PSD (Class I; Level of Evidence B)

Middle-aged and young-elderly: There is reasonable evidence that... lowering blood pressure can be useful for the prevention of late-life dementia (Class Ila; Level of Evidence B)

Adults $>80$ years: The usefulness of lowering blood pressure... for the prevention of dementia is not well established (Class Ilb; Level of Evidence B)

The effectiveness of treating diabetes/hyperglycemia for the prevention of dementia is not well established (Class IIb; Level of Evidence C)

The usefulness of treatment of hyperlipidemia for prevention of dementia is uncertain (Class Ilb; Level of Evidence C)

A Mediterranean-type dietary pattern has been associated with less cognitive decline in several studies and may be reasonable (Class Illb; Level of Evidence B)

Vitamin supplementation is not proven to improve cognitive function, even if homocysteine levels have been positively influenced, and its usefulness is not well established (Class Illb; Level of Evidence B)

Physical activity might be considered for the prevention of cognitive impairment (Class Ilb; Level of Evidence B), but the usefulness of other lifestyle or vitamin interventions is uncertain (Class Ilb; Level of Evidence B)

\section{The effectiveness of antiaggregant therapy for $\mathrm{VCl}$ is not well established (Class Illb; Level of Evidence B) Donepezil can be useful for cognitive enhancement in patients with $\mathrm{VaD}$ (Class Ila; Level of Evidence A)}

Galantamine can be beneficial for patients with mixed Alzheimer disease/VaD (Class lla; Level of Evidence A)

The benefits of rivastigmine are not well established in VaD (Class Illb; Level of Evidence A)

NMDA antagonists The benefits of memantine are not well established in VaD (Class Ilb; Level of Evidence A)

Abbreviations: $\mathrm{AHA}=$ American Heart Association; $\mathrm{ASA}=$ American Stroke Association; PSD = poststroke depression; VaD; vascular dementia; $\mathrm{VCl}=$ vascular cognitive impairment.

adapted from "Vascular contributions to cognitive impairment and dementia: A statement for healthcare professionals from the American Heart Association/American Stroke Association." ${ }^{4}$

Drug Association-approved florbetapir tracer or measuring CSF levels of amyloid beta, phosphorylated tau, and total tau can be helpful in sorting out the underlying contribution of $\mathrm{AD}$, even if their specificity in the setting of VCI remains unclear. Similarly, a dopamine transporter scan using SPECT imaging may help determine whether Parkinson disease or Lewy body dementia is contributing to the clinical picture. Given the lack of therapeutic modalities for neurodegenerative causes of dementia, the extent of the diagnostic workup raises ethical and financial questions, and patients and families need to understand the limitations of current therapies and the objectives of in-depth clinical evaluations.

Prevention and treatment of VCI As noted above, risk factor control and lifestyle modifications that include improved diet and greater physical activity are likely to be key in the prevention of vascular disease and greater SCVD that may result in VCI. However, most existing data come from observational studies, and randomized controlled trials (RCTs) have focused on only a few risk factors. Clinical trials generally support the idea that treatment of hypertension is beneficial, but a number of limitations to these trials have been noted, including insensitive cognitive outcome measures, bias from selective loss to follow-up, short follow-up times, and limited statistical power. More definitive studies, such as the ongoing Systolic Blood Pressure Intervention Trial (SPRINT), are ongoing. The benefits of treating other common 
potentially modifiable risk factors are likewise not established. Similar to hypertension, diabetes and impaired glucose metabolism are associated with cognitive decline and dementia, but RCTs have not shown a benefit to treatment. The beneficial effects of lipid lowering have also not been proven in a well-designed RCT, although there is some evidence that treatment with statins may improve cognitive outcomes. ${ }^{29}$ Lifestyle modifications such as the Mediterranean diet and physical exercise also show some benefit for the prevention of MCI and AD in observational studies, but a link with VCI is yet to be established fully and RCT evidence is lacking. The table summarizes recommendations in the AHA/ASA statement on VCI regarding a number of modifiable and lifestyle factors as well as pharmacologic interventions that have been studied to date. ${ }^{4}$ Several of the cholinesterase inhibitors have been found to improve cognitive performance in pure and "mixed" $\mathrm{VaD}$, but studies examining the interaction of such treatments with markers of SCVD are lacking.

\section{REFERENCES}

1. Launer LJ, Ross GW, Petrovitch H, et al. Midlife blood pressure and dementia: the Honolulu-Asia aging study. Neurobiol Aging 2000;21:49-55.

2. Luchsinger JA, Tang MX, Stern Y, Shea S, Mayeux R. Diabetes mellitus and risk of Alzheimer's disease and dementia with stroke in a multiethnic cohort. Am J Epidemiol 2001;154:635-641.

3. Yaffe K, Haan M, Blackwell T, Cherkasova E, Whitmer RA, West N. Metabolic syndrome and cognitive decline in elderly Latinos: findings from the Sacramento Area Latino Study of Aging study. J Am Geriatr Soc 2007;55:758-762.

4. Gorelick PB, Scuteri A, Black SE, et al. Vascular contributions to cognitive impairment and dementia: a statement for healthcare professionals from the american heart association/american stroke association. Stroke 2011;42:2672-2713.

5. McKhann GM, Knopman DS, Chertkow H, et al. The diagnosis of dementia due to Alzheimer's disease: recommendations from the National Institute on Aging-Alzheimer's Association workgroups on diagnostic guidelines for Alzheimer's disease. Alzheimers Dement 2011;7:263-269.

6. Debette S, Markus HS. The clinical importance of white matter hyperintensities on brain magnetic resonance imaging: systematic review and meta-analysis. BMJ 2010;341:c3666.

7. Looi JC, Sachdev PS. Differentiation of vascular dementia from AD on neuropsychological tests. Neurology 1999;53:670-678.

8. Reed BR, Mungas DM, Kramer JH, et al. Profiles of neuropsychological impairment in autopsydefined Alzheimer's disease and cerebrovascular disease. Brain 2007;130:731-739.

9. Pendlebury ST, Mariz J, Bull L, Mehta Z, Rothwell PM. MoCA, ACE-R, and MMSE versus the National Institute of Neurological Disorders and Stroke-Canadian Stroke Network Vascular Cognitive Impairment Harmonization Standards Neuropsychological Battery after TIA and stroke. Stroke 2012; 43:464-469.

10. Hachinski V, Iadecola C, Petersen RC, et al. National Institute of Neurological Disorders and StrokeCanadian Stroke Network vascular cognitive impairment harmonization standards. Stroke 2006;37: 2220-2241.

11. Pendlebury ST, Rothwell PM. Prevalence, incidence, and factors associated with pre-stroke and poststroke dementia: a systematic review and meta-analysis. The Lancet Neurol 2009;8:1006-1018.

12. Ivan CS, Seshadri S, Beiser A, et al. Dementia after stroke: the Framingham Study. Stroke 2004;35: 1264-1268.

13. Sachdev P, Kalaria R, O'Brien J, et al. Diagnostic criteria for vascular cognitive disorders: a VASCOG statement. Alzheimer Dis Assoc Disord 2014;28:206-218.

14. Jacova C, Pearce LA, Costello R, et al. Cognitive impairment in lacunar strokes: the SPS3 trial. Ann Neurol 2012;72:351-362.

15. Dubois B, Slachevsky A, Litvan I, Pillon B. The FAB: a Frontal Assessment Battery at bedside. Neurology 2000;55:1621-1626.

16. Savva GM, Wharton SB, Ince PG, et al. Age, neuropathology, and dementia. N Engl J Med 2009; 360:2302-2309.

17. Moroney JT, Bagiella E, Desmond DW, et al. Meta-analysis of the Hachinski Ischemic Score in pathologically verified dementias. Neurology 1997;49:1096-1105.

18. Reed BR, Mungas DM, Kramer JH, et al. Clinical and neuropsychological features in autopsy-defined vascular dementia. Clin Neuropsychol 2004;18:63-74.

19. Markus HS, Martin RJ, Simpson MA, et al. Diagnostic strategies in CADASIL. Neurology 2002;59: $1134-1138$. 
20. Park JH, Seo SW, Kim C, et al. Pathogenesis of cerebral microbleeds: in vivo imaging of amyloid and subcortical ischemic small vessel disease in 226 individuals with cognitive impairment. Ann Neurol 2013;73:584-593.

21. Vermeer SE, Prins ND, den Heijer T, Hofman A, Koudstaal PJ, Breteler MM. Silent brain infarcts and the risk of dementia and cognitive decline. N Engl J Med 2003;348:1215-1222.

22. Kwan LT, Reed BR, Eberling JL, et al. Effects of subcortical cerebral infarction on cortical glucose metabolism and cognitive function. Arch Neurol 1999;56:809-814.

23. Gutierrez J, Rundek T, Ekind MS, Sacco RL, Wright CB. Perivascular spaces are associated with atherosclerosis: an insight from the Northern Manhattan Study. AJNR Am J Neuroradiol 2013;34: 1711-1716.

24. Pullicino PM, Miller LL, Alexandrov AV, Ostrow PT. Infraputaminal 'lacunes'. Clinical and pathological correlations. Stroke 1995;26:1598-1602.

25. Wardlaw JM, Smith EE, Biessels GJ, et al. Neuroimaging standards for research into small vessel disease and its contribution to ageing and neurodegeneration. Lancet Neurol 2013;12:822-838.

26. Arvanitakis Z, Leurgans SE, Barnes LL, Bennett DA, Schneider JA. Microinfarct pathology, dementia, and cognitive systems. Stroke 2011;42:722-727.

27. Sullivan P, Pary R, Telang F, Rifai AH, Zubenko GS. Risk factors for white matter changes detected by magnetic resonance imaging in the elderly. Stroke 1990;21:1424-1428.

28. Brickman AM, Provenzano FA, Muraskin J, et al. Regional white matter hyperintensity volume, not hippocampal atrophy, predicts incident Alzheimer disease in the community. Arch Neurol 2012;69: $1621-1627$.

29. Wong WB, Lin VW, Boudreau D, Devine EB. Statins in the prevention of dementia and Alzheimer's disease: a meta-analysis of observational studies and an assessment of confounding. Pharmacoepidemiol Drug Saf 2013;22:345-358.

\section{STUDY FUNDING}

No targeted funding reported.

\section{DISCLOSURES}

C.B. Wright serves on the NOCIP data safety monitoring board; receives publishing royalties from $U_{p T o D a t e}$; serves as a consultant for Merck; and receives research support from the American Heart Association (ASA/Bugher Foundation Center project 14BFSC17690003) and the NIH (K02 NS 059729, R01 HL 108623, R37 NS 29998). A. Flores reports no disclosures. Full disclosure form information provided by the authors is available with the full text of this article at Neurology.org/cp.

\section{Related articles from AAN physician and patient resources}

\section{Neurology ${ }^{\circledR} \quad-\quad$ Neurology.org}

Effects of vascular risk factors and $A P O E \in 4$ on white matter integrity and cognitive decline March 17, 2015;84:1128-1135.

Multiple or mixed cerebral microbleeds and dementia in patients with vascular risk factors August 12, 2014;83:646-653.

\section{Continuum ${ }^{\circledR}$ - ContinuumJournal.com}

Understanding and Treating Vascular Cognitive Impairment April 2013;19:425-437.

\section{Neurology Today ${ }^{\circledR} \quad$ - Neurotodayonline.com}

The Connection Between Alzheimer's and Small-Vessel Disease

June 19, 2014,14:1,9-11. 